\title{
Production of Bisphenol A (BPA) By Green Technology
}

\author{
Ibrahim Altuwair* \\ Department of Engineering \& Applied Science, Memorial University, Canada
}

Submission: January 11, 2018; Published: April 10, 2018

*Corresponding author: Ibrahim Altuwair, Faculty of Engineering \& Applied Science, Memorial University, St. John's, NL, Canada,

Tel: +1709 770 4044,Email: iaa134@mun.ca

\begin{abstract}
The literatures and researches show that the reaction between phenol and acetone is too slow in the absence of catalyst, but it proceeds with formation of Bisphenol A (BPA) in the presence of acidic catalyst or any strong acid [1]. Although the BPA formation depends on the molar ratio of reactants (acetone and phenol), using a different solvents or catalysts will not deny the effect of the BPA on human health. It is only enhancing the reaction ratio between phenol and acetone and improves the selectivity. There are different catalysts and solvents have been tested and their effects on the process and BPA yields were evaluated. However, as the reaction proceeded to increase the water concentration, the inhibition effect of water reduces the reaction rate and gives low yield. This will not eliminate the impacts of BPA directly or indirectly on human health, it is only increased the quality of the product.
\end{abstract}

Keywords: Bisphenol A; Process; Reaction; Acetone; Catalyst; Concentration

Abbreviations: DCM: Dichloromethane; DOE: Design Of Experiment; PTSA : P Toluene Sulfonic Acid; DVB: Divinyl Benzene; SCF: Supercritical Fluid

\section{Introduction}

\section{Bisphenol A production process}

The aim of the chapter is to give an overview of the BPA production methods.

\section{Preparation of Bisphenol A}

The BPA synthesis including mechanisms, possible reactions, by-products, and order of reaction.

The acid catalyzed condensation of acetone with two moles of phenol is the first process for forming BPA, (Figure 1).

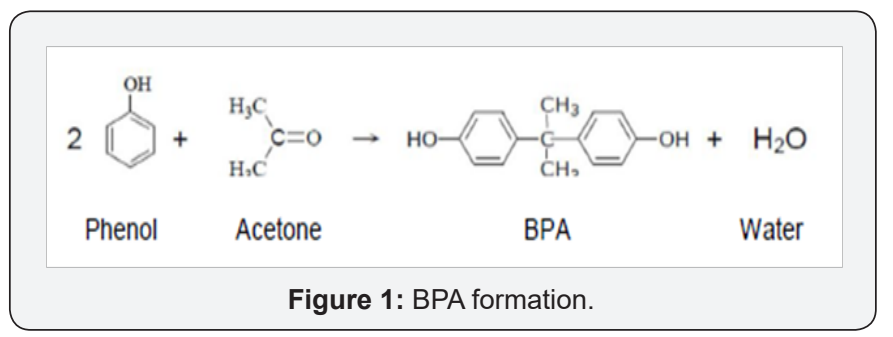

The heat of reaction, for reagents and products in their natural physical state at $25^{\circ} \mathrm{C}$, is calculated from heats of formation as $19 \mathrm{kcal} / \mathrm{mol}$ and a molar ration of $1: 2$ acetone to phenol, is considered in the first stage of the research, in the presence of concentrated hydrochloric acid at room temperature. There was a claim of the present of small amount of water (10\%) or less in the reaction mixture increases the reaction rate of the mixture catalyzed by hydrochloric acid [2]. Others suggest that processing the reaction by ion-exchange resin (sulfonic acid) modified with alkyl-SH groups enhance the reaction and decrease the amount of water $5 \%$ by weight in the mixture which decreases the yield

of BPA. However, it was suggested by Jerabek et al. that to reduce the effect of water, dehydration by various water-binding agents (such as Calcium Chloride or phenyl acetate) or by azeotropic distillation can be used.

This reaction proceeds with an electrophilic attack of the proton from the acidic catalyst on the molecule of acetone. During this step, producing by-products or "impurities" might change the mechanism of the reaction by produce undesirable products as a result of acetone - acetone reaction or Dimerization. Thus, Mesityl oxide is formed and increasing the number of impurities that lead to further phenolic reaction and the process becomes more complicated. Therefore more effects on human health are expected due to phenolic impacts. 
The most industrially used processes for making BPA in the United States and Western Europe are the acetone-phenol Process, There are different methods of producing Bisphenol A.

\section{Conventional Methods}

This method based on the type of the catalyst and solvent. It is also called Hydrogen Chloride-catalyzed process. The first production of BPA was made by Russian scientist, Dianin, in 1891. He used Hydrochloric acid to accelerate the reaction since it is hard to form BPA without strong acid. However, the process of obtaining BPA is also depending on the phenol to ketone (acetone) reaction ratio. Thus, there will be excessive phenol, approximately a ratio of (3-30):1 to ensure the reaction is processed (WO 2005/030687, 2005). Although the reaction gives high efficiency, the conventional methods of BPA production does not provide approximately high selectivity of the reaction of phenol with acetone and the required purity of the desired product. It also appeared that it is impossible to achieve the equilibrium state for some by-product of the process therefore the process cannot be performed for long time without production loss. In addition, because of the low concentration of BPA, high energy consumption will be resulting from the process.

\section{Alternative Methods}

There are alternative methods to produce BPA by using different catalysts with/without promoters. This method is also known as the resin-catalyzed process. It gives better results in terms of selectivity, and purity of product. One of the catalyst that been used such as exchanger resin also improves the production of BPA. In addition, there are other catalysts have been used and modified to increase the BPA concentration and improve the phenol and acetone reaction, for instance, sulfonated ion exchange resin, DVB divinyl benzene, and polystyrene. Although the formation of BPA is improving continuously, still there is no answer for the variation in ratio of phenol to acetone.

\section{Parameters}

The selection of parameters depends on the specific compound. There are several important parameters need to be taking into account during the process:

1) Temperature

2) Time

3) Catalyst

According to Arrhenius equation, the results obtained were temperature dependence, for the water adsorption coefficient KW. For the adsorption coefficient of acetone and phenol increased with the temperature, although, the rate constant of reaction is increased with temperature, the energy of between acetone and phenol is weaker (Figure 2).

$$
K=1470 \operatorname{Exp}(-59900 / R T)\left[\mathrm{m}^{3} \mathrm{~kg}^{-1} \mathrm{~s}^{-1}\right]
$$

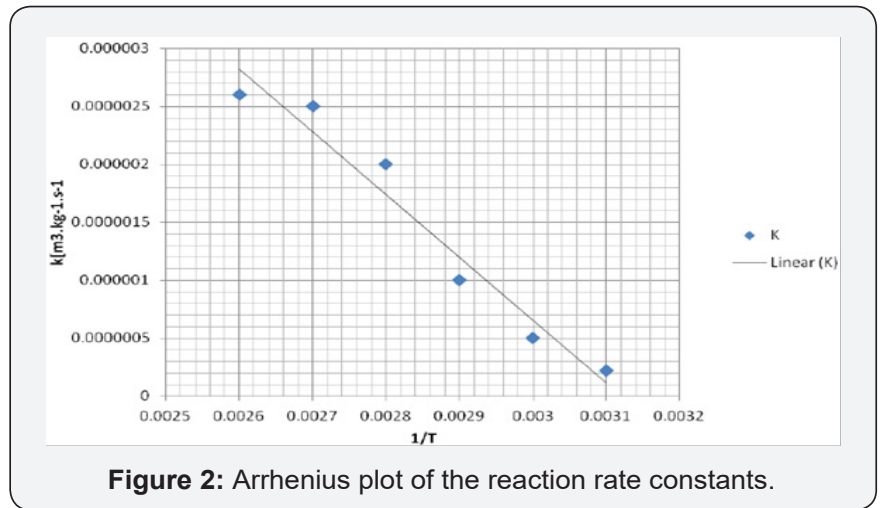

This showed that higher energy is needed to complete the formation of BPA due to the slow processing mixture.

\section{Experimental Section}

\section{Experimental Procedures}

P-Toluene sulfonic acid, acetone, and phenol were purchased from Sigma-Aldrich Company. An appropriate amount of an aqueous solution of chloroform was added to the solution at room temperature. Synthesis of bisphenol A(BPA) was carried out in the liquid phase under atmospheric pressure and $600 \mathrm{C}$ in $1000 \mathrm{ml}$ two-neck round bottom flask equipped with a condenser and a magnetic stirrer. An 8.6g of PTSA catalyst put into the reactor with $23.5 \mathrm{~g}$ of phenol. Then, $2.9 \mathrm{~g}$ of acetone was added by a micro- syringe to start the bisphenol a synthesis. Yield was determined by a column chromatography. Furthermore, vacuuming the sample was applied after separation process to purify the final product from the residual solvents. Weights were measured before and after evaporation.

Characterization: Bisphenol A monomer synthesis from the condensation reaction of phenol with acetone will be analyzed by using Column Chromatography, NMR, GC-MS techniques. Spectroscopic analysis showed that BPA monomer was present in the product although significant amounts of compound formed during the reaction and residual starting material were still present.

After sampling and cooling down the samples at $0{ }^{\circ} \mathrm{C}$, evaporation was applied by using rotary evaporator for 10-20 minutes at $40-60{ }^{\circ} \mathrm{C}$. HNMR spectrum at $400 \mathrm{MHZ}$ was measured by a Bruker DMX500 with the following condition; Spinning rate $5 \mathrm{KHZ}$, Constant time $1.75 \mathrm{msec}$, interval $4 \mathrm{sec}$, accumulation 500 times, and glycine as an external standard of chemical shift. Before the measurement, the sample was cooled at

$-20^{\circ} \mathrm{C}$.

Materials and equipments: Phenol and acetone as the main reactants of reaction brought from Sigma-Aldrich Company. P-toluene sulfonic acid-PTSA is available in the chemistry lab with chloroform, Methanol, Dichloromethane DCM, acetonenitrile, $\left(\mathrm{CDCl}_{3}\right.$, DMSO were used as deuterated solvents for NMR) Silica gel and Sand were also available in the lab. 
$250 \& 1000 \mathrm{ml}$ two neck round bottom flask were used, rotary evaporator R-114, water bath B-480\&B-490RE Buchi, condenser, thermometer.

Process Selection: Condensation of phenol with acetone in the presence of p-Toluene sulfonic acid (PTSA) as a catalyst.

Process Description: Acetone and excess phenol are reacted at a temperature of $600 \mathrm{C}$ and atmosphere pressure by condensation in $\mathrm{p}$-Toluene Sulfonic Acid catalyzed reactor system to produce $\mathrm{p}, \mathrm{p}$-bisphenol A, water and various by-products are also formed in the reaction process. The crude distillation or chromatography column helps to remove water and unreacted acetone from the reactor effluent [3]. Acetone and un reacted materials will be sent to rotary evaporation process at 50-60 ${ }^{\circ} \mathrm{C}$ for more purification then mother liquor from purification system is cooled down at -20 .

\section{Equipments Used:}
1) Reactor
2) Oil bath
3) Condenser
4) Heater
5) Chromatography Column (CC)
6) Rotary Evaporator

\section{Description}

A. Components of a control System

The system is divided into the following components:

1) Process

2) Measuring element

3) Controller

B. Controlling System Used:

1) Temperature Control

2) Stirring control

3) Pressure Control

Temperature Control: It is desired to maintain the temperature in the reactor by using a controller. If the measured temperature differs from the desired temperature, the controller senses the changes and modify at the required temperature.

Stirrer control: Stirring can be control by using magnetic stirrer .In this experiment, IEC Magnetic stirrer was used with CAT No.: CH2090-001 STIR Input: 220/240V.AC.50/60Hz.4AMP.

Pressure control: The pressure maintains at the atmospheric pressure. However, low pressure was used for some processes $[4,5]$.
Preparation Method: The experiment carried out in the chemistry lab based on the reaction of phenol- acetone reaction and also the reaction will be tested in different ways. Firstly, the reaction of acetone and phenol will be carried out. Secondly, the homogeneous catalyst will be added. Thirdly, the reaction will be processed in the presence of supercritical carbon dioxide, $\mathrm{scCO}_{2}$. The report will cover the first and second parts.

The main goal of the project is to use supercritical carbon dioxide as a reaction medium for the production of BPA and also for separation purposes to remove undesired products easily. Minimizing the effect of the undesired products on the BPA formation by using SCF will be another target for the study as an advantage of using green technology for BPA production and reduce the chemicals that emitted into the atmosphere and polluted the environment. This technique will be applied after synthesizing BPA by different methods to investigate and evaluate their results with/without using $\mathrm{ScCO}_{2}$ technology.

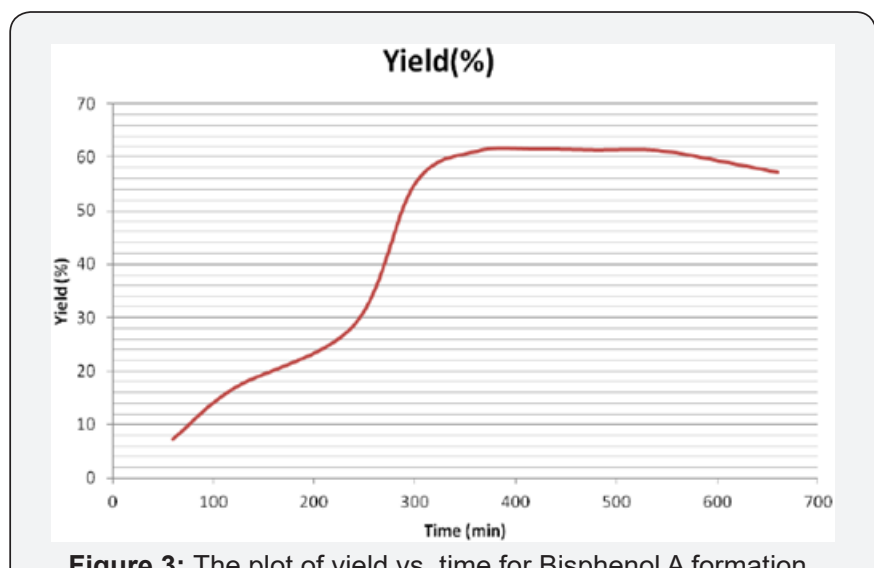

Figure 3: The plot of yield vs. time for Bisphenol A formation.

Table 1: Experimental Data.

\begin{tabular}{|c|c|c|c|}
\hline Sample & T(min) & Weight(g) & Yield (\%) \\
\hline 1 & 60 & 16.45 & 7.21 \\
\hline 2 & 120 & 38.12 & 16.69 \\
\hline 3 & 240 & 65.42 & 28.65 \\
\hline 4 & 300 & 125.64 & 55.04 \\
\hline 5 & 360 & 139.5 & 61.1 \\
\hline 6 & 420 & 140.5 & 61.54 \\
\hline 7 & 480 & 140.12 & 61.37 \\
\hline 8 & 540 & 140.02 & 61.33 \\
\hline 9 & 600 & 135.62 & 59.4 \\
\hline 10 & 660 & 130.54 & 57.18 \\
\hline
\end{tabular}

Experimental results and discussion: In order to understand the reaction mechanism, several experiments were conducted in the chemistry lab. These experiments were carried out at 60-800C for all over variables, such as concentration of catalyst and reactants fixed at maximum yield level. At the end of the experiment, the reaction mixture was cooled down at room temperature and then stored at 20 after dried and weighed. The 
yield of the reaction mixture was calculated for each experiment. The plot of yield vs. time is shown in Figure 3.

$$
X=1-(1+0 / 03 t)^{-2 / 0}
$$

Where, $\mathrm{X}$ : is fraction conversion of limiting component (mol/ mol) (Table 1).

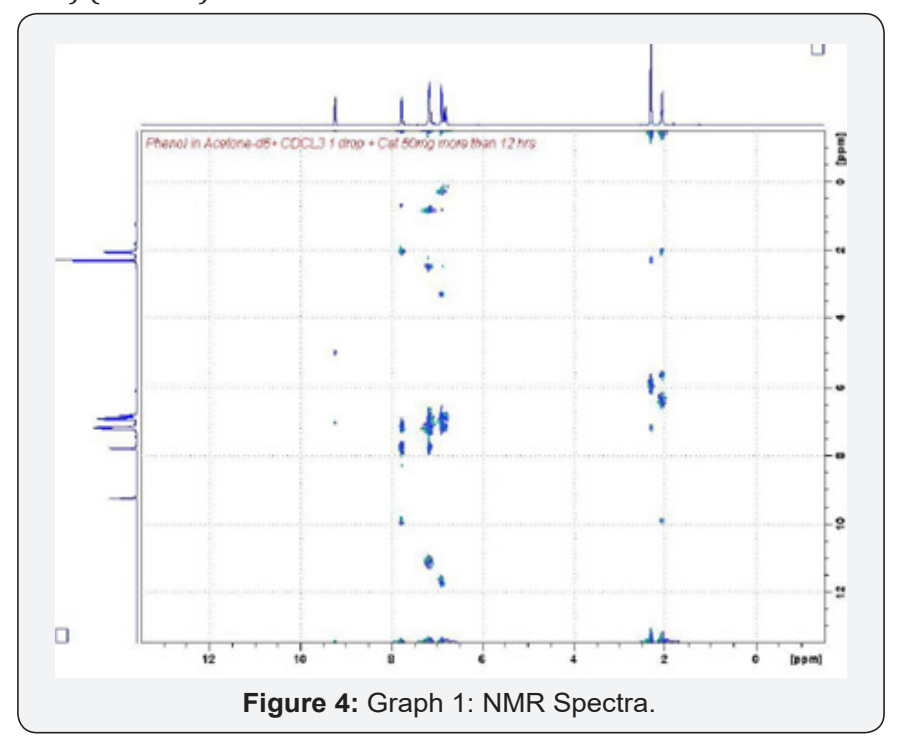

The graph is in a good agreement with literature data, this

Table 2: Data Analysis.

\begin{tabular}{|c|c|c|c|c|c|c|c|c|c|}
\hline Time & A(Phenol) & B(Phenol) & C(BPA) & D(BPA) & (C+D)/8 & (A+B)/5 & Ratio & Conversion & \% \\
\hline 0 & 50 & 50 & 0 & 0 & 0 & 20 & 0 & 0 & 0 \\
\hline 2 & 10.25 & 17.98 & 1.91 & 2.11 & 0.5025 & 5.646 & 0.089001 & 0.075553 & 37.77627 \\
\hline 4 & 13.34 & 22.36 & 6.95 & 6.68 & 1.70375 & 7.14 & 0.23862 & 0.161531 & 80.76558 \\
\hline 6 & 11.91 & 24.45 & 9.23 & 10.37 & 2.45 & 7.272 & 0.336909 & 0.201282 & 100.6408 \\
\hline 8 & 15.34 & 30 & 13.14 & 9.39 & 2.81625 & 9.068 & 0.31057 & 0.191575 & 95.78756 \\
\hline 10 & 19.65 & 37.39 & 14.03 & 14.01 & 3.505 & 11.408 & 0.307241 & 0.190303 & 95.15148 \\
\hline
\end{tabular}

\section{Analysis}

The samples were analyzed by HNMR (Gyro and Flip Machines). Gyro system was used to analyze the structure of the product where flip was used for kinetic reaction purpose. All procedures and results of the analysis are described in the following sections. The graph shows the total acetone converted to product and time needed for completion. It is very clear that after 6 hours of reaction, the whole acetone converted to products.

\section{Design of Experiment (DOE)}

The following section will be focus on the experimental design to investigate and evaluate the individual and interaction effect. Using different techniques to deal with noise in an experiment, and make a good decision for the chemical process. To design an experiment by using engineering method or program, the following steps need to be investigated:

1) Response Variables plot was generated based on experimental data (Figure 4) (Table 2).

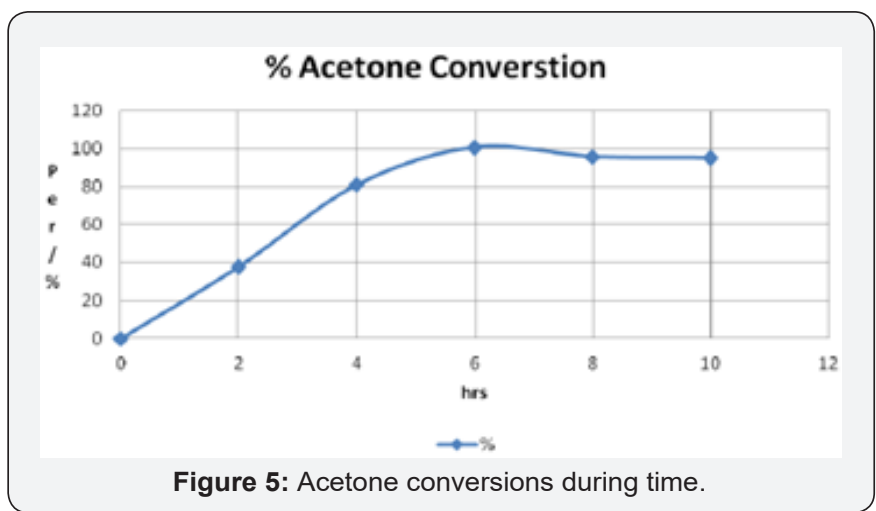

Conversion $\%=\mathrm{R} /(1+2 \mathrm{R})$

Where,

R: Ratio

Ratio $=F / G$

$\mathrm{G}:(A+B) / 5$

F: $(C+D) / 8$

(Figure 5)
2) Factors
3) Randomization
4) Repetition and Replication

Response Variables: Response Variables are the variables that measured and the object of the characterization or optimization (Y's). However, defining the response will be the hardest part to design an experiment. To solve this problem, several questions have to be answer such as:
a. How will the results be analyzed
b. How good is the measurement system?
c. What are the baseline mean and standard deviation?

Factors: They are variables which controlled or varied in a systematic way during the experiment. These two steps are important to design the experiment and evaluate or investigate the factors effect on the process. 
The purpose of using an experimental design is to deal with different factors and make a good decision for an optimal production process. Before starting the design of experiment DOE, pre-experimental plan is recommended.

\section{a. Experimental Design Procedures}

Planning: Before starting the computer program, preexperimental planning needs to be done. $t \backslash$ The preparation require before beginning experimentation depends on your problem. For example, you need to determine what influence factors are, what processing condition influence, thus careful planning can help to avoid problem that can occur during the execution of the experimental plan.

Processing Characterization (Screening): In many process development and manufacturing applications, potentially influential variables are numerous. Screening reduces the number of variables by identifying the key variables that affect product quality. This reduction allows focusing on the really important variables. Screening may also suggest the best or optimal setting for these factors. Then you can use optimization methods to determine the best settings and define the nature of the curvature. Screening test by running a relatively small number of tests to isolate the most important factors.

Optimization: (optimize a process/product): The method of optimization helps to determine the best settings and define the nature of the curvature. The optimal values for experimental factors depend on the process objectives. The objective of this experimental is to reduce by-products and maximize the BPA Yield.

Randomization: It can be done in many ways

a. Run the treatment combination in random order

b. Assign experimental units to the treatment combinations randomly

c. The advantage of randomization is to average out the effect of the extraneous factors (called noise) that may be present but were not controlled or measured during the experiment.

d. Spread the effects of the noise across all runs

e. These extraneous factors (noise) cause unexplained variation in the response variable (Yield).

Performance Equation

$$
\begin{aligned}
& r A V=\frac{d_{n} A}{d_{t}} \\
& n_{A}=V^{*} C_{A} \\
& r A V=V \frac{d C A}{d t}
\end{aligned}
$$

Then,

$$
r_{A}=\frac{d C A}{d t}
$$

\begin{tabular}{|c|c|c|c|c|}
\hline Run Order & Std Order & Temperature & Time & Concentration \\
\hline 1 & 15 & -1 & -1 & -1 \\
\hline 2 & 3 & -1 & -1 & 1 \\
\hline 3 & 13 & -1 & 1 & -1 \\
\hline 4 & 18 & -1 & 1 & 1 \\
\hline 5 & 10 & 1 & -1 & -1 \\
\hline 6 & 5 & 1 & -1 & 1 \\
\hline 7 & 9 & 1 & 1 & -1 \\
\hline 8 & 14 & 1 & 1 & 1 \\
\hline 9 & 7 & $\alpha$ & 0 & 0 \\
\hline 10 & 16 & $-\alpha$ & 0 & 0 \\
\hline 11 & 4 & 0 & $\alpha$ & 0 \\
\hline 12 & 11 & 0 & $-\alpha$ & 0 \\
\hline 13 & 2 & 0 & 0 & $\alpha$ \\
\hline 14 & 12 & 0 & 0 & $-\alpha$ \\
\hline 15 & 1 & 0 & 0 & 0 \\
\hline 16 & 8 & 0 & 0 & 0 \\
\hline 17 & 17 & 0 & 0 & 0 \\
\hline 18 & 20 & 0 & 0 & 0 \\
\hline 19 & 19 & 0 & 0 & 0 \\
\hline 20 & 6 & 0 & 0 & 0 \\
\hline
\end{tabular}

\section{(Table 3,4) (Figure 6)}

Table 3: shows the randomization method.

Table 4: The production rate.

\begin{tabular}{|c|c|c|c|c|c|}
\hline Time & CA0 & CA & (CA/CA0) & $\operatorname{In(CA/CA0)~}$ & Kt \\
\hline 1 & 0.1 & 0.09978 & 0.997802 & -0.0022 & -0.0022 \\
\hline 2 & 0.2 & 0.199122 & 0.99561 & -0.0044 & -0.0044 \\
\hline 3 & 0.3 & 0.298027 & 0.993422 & -0.0066 & -0.0066 \\
\hline 4 & 0.4 & 0.396495 & 0.991239 & -0.0088 & -0.0088 \\
\hline 5 & 0.5 & 0.49453 & 0.98906 & -0.011 & -0.011 \\
\hline 6 & 0.6 & 0.592132 & 0.986887 & -0.0132 & -0.0132 \\
\hline 7 & 0.7 & 0.689303 & 0.984718 & -0.0154 & -0.0154 \\
\hline 8 & 0.8 & 0.786043 & 0.982554 & -0.0176 & -0.0176 \\
\hline 9 & 0.9 & 0.882355 & 0.980395 & -0.0198 & -0.0198 \\
\hline 10 & 1 & 0.97824 & 0.97824 & -0.022 & -0.022 \\
\hline
\end{tabular}

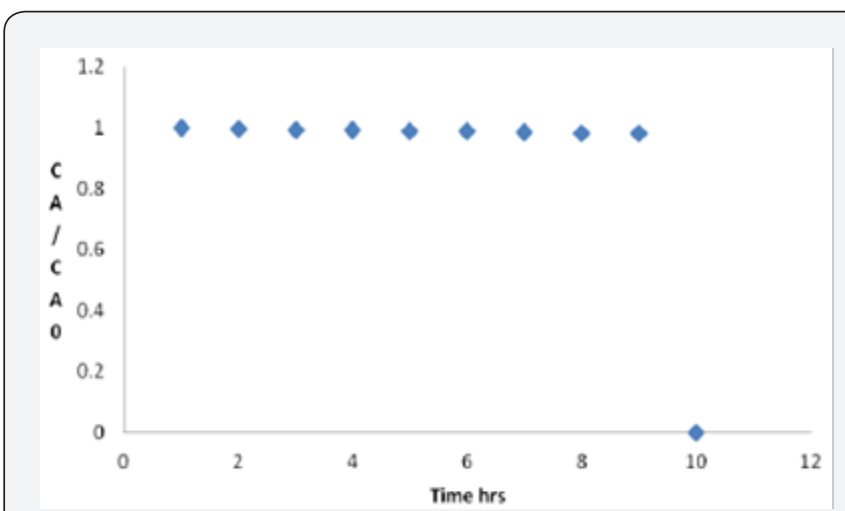

Figure 6: The graph of concentration over time. 
Minitab Program
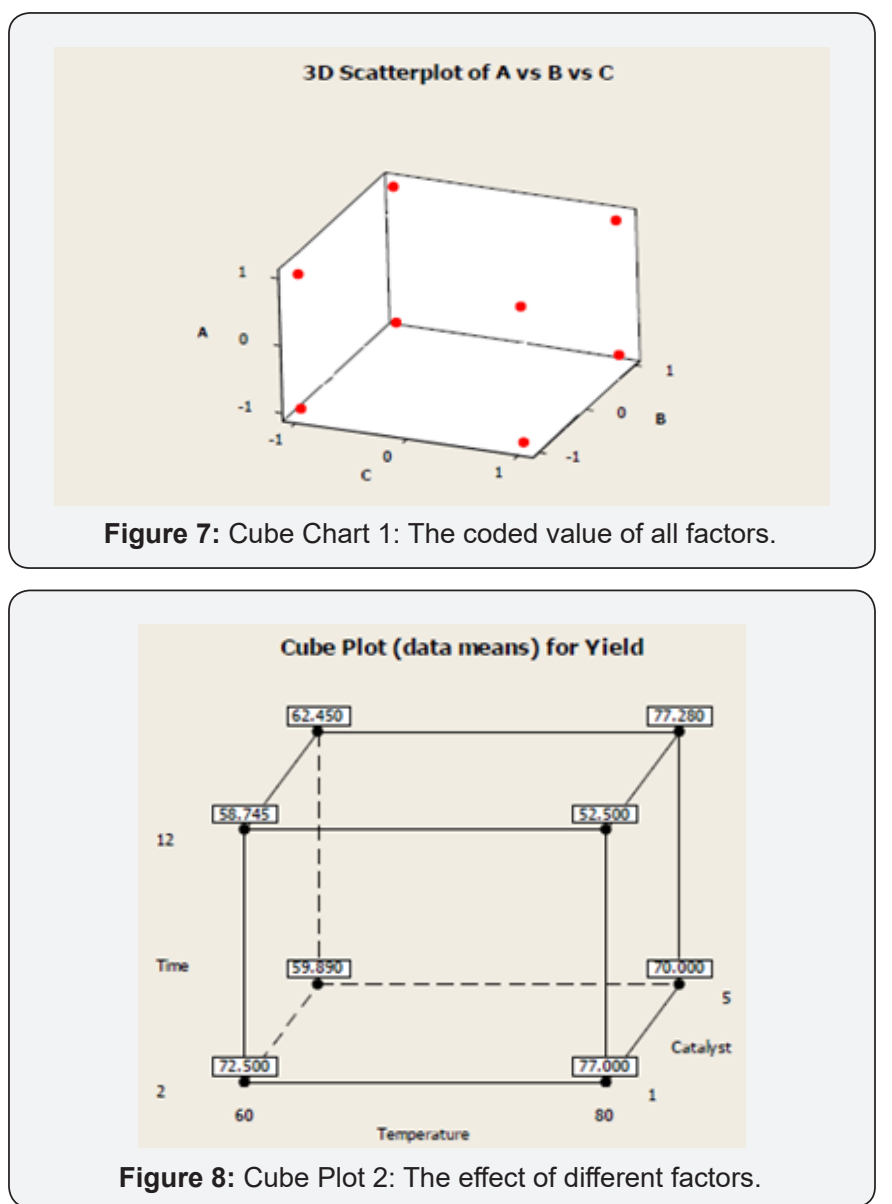

The below cube chart shows the coded value of all factors from high to low values (1to-1) (Figure 7,8).

The Cube Chart, the effect of different factors can be calculated by applying the following equation:

$$
\text { Effect }=[(+Y / n)-(-Y / n)]
$$

\section{Interaction plot}

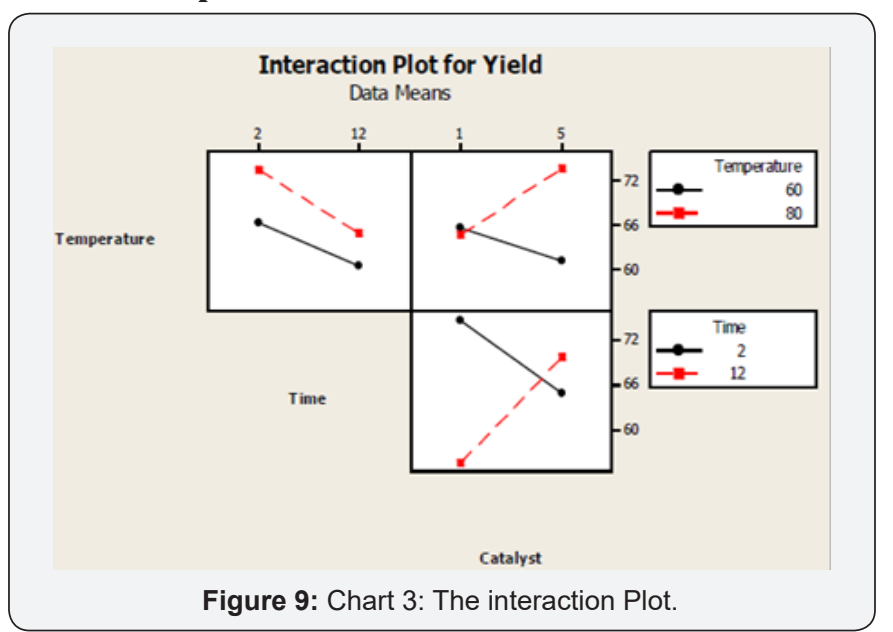

The following graphs show the interaction effect of the different parameters on the process. The interaction between catalyst and time has the greatest or significant effect on the process followed by the interaction effect of catalyst and temperature then time and temperature interaction effect.

By using the Minitab Programm, it is easy to evaluate the significant factors with significant effects on the reaction process and eliminate all factors without real effect on the process. Also the program minimize the time that will be spent during the reaction and focus only on the significant factors which will save time and money (Figure 9).

\section{Results and Discussion}

Table 5: General Linear Model: Yield versus Temperature, Time.

\begin{tabular}{|c|c|c|c|c|c|c|}
\hline Source & DF & Seq SS & Adj SS & Adj MS & F & P \\
\hline Temperature & 1 & 777.7 & 777.7 & 777.7 & 1.94 & 0.201 \\
\hline Time & 1 & 40.2 & 40.2 & 40.2 & 0.1 & 0.76 \\
\hline
\end{tabular}

General linear model: Yield versus temperature, time (Table 5)

Factorial fit: Yield versus temperature, time, catalyst (Table 6): Estimated Effects and Coefficients for Yield (coded units)

Table 6: Estimated Effects and Coefficients for Yield (coded units).

\begin{tabular}{|c|c|c|c|}
\hline Term & Effect & CoefT & P \\
\hline Constant & 47.073 & 9.4 & 0 \\
\hline Temperature & -13.944 & -6.972 & 0.201 \\
\hline Time & 3.171 & 1.586 & 0.76 \\
\hline Catalyst & -11.146 & -5.573 & 0.298 \\
\hline Temperature*Time & -0.969 & -0.484 & 0.925 \\
\hline Temperature*Catalyst & -12.056 & -6.028 & 0.263 \\
\hline Time*Catalyst & 18.829 & 9.414 & 0.097 \\
\hline Temperature*Time*Catalyst & -17.031 & -8.516 & 0.127 \\
\hline
\end{tabular}

Al these values demonstrate the interaction effects on the process such as Temperature and catalyst interaction that have significant affects by high effect value and lowest P-value.

\section{Evaluation}

The mechanism of bisphenol reaction from industrial starting material was studied. The obtained yield -time relationship was obtained .It was found that at about $62 \%$ can be obtained after 6 hours of reaction.

The experimental design detects the effects of different factors on the process. In addition, the experimental data shows the reaction rate of the starting materials and final products which is in agreement with the HNMR spectra results even though there were some experimental errors due to the equipments and measurement errors. In addition, designing the experiment by DOE presents the experiment by engineering way and save time and money for future research. 


\section{Drawbacks}

Bisphenol A-BPA production process is continuously improved by different ways even by manipulating parameters. However, there's no answer to stoichiometric ration acetone to phenol variation from $1-2$ to $1-10$ or 30 , which add extra processing required during purification. Thus, the reaction becomes more complicated (Figure 10).

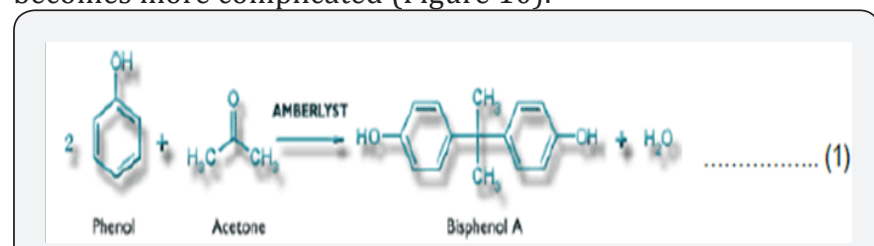

Figure 10: Phenol and Acetone Reaction by ion-exchange resin

The reaction is the industrial reaction to produce BPA by using ion exchange resin. The Reaction shows that swollen ion exchange resin with $\mathrm{SO}_{3} \mathrm{H}$ group attacks the aromatic ring to form BPA. Firstly, $\mathrm{SO}_{3} \mathrm{H}$ group will react with carbonyl group (acetone) by less than completing proton $\mathrm{H}+$ and then acetone will react with phenol to give the final product of BPA, the two aromatic rings shown on the BPA structure describe the need of excessive phenol to react with one acetone in order to produce BPA and water. However, producing water decreases the catalytic activity of the resin. As water spreads towards the outlet, the acetone conversion decreases [6].

This accumulation of water prevents a long-term operation of the process; therefore, to stabilize the operation process, water must be removed. Producing water during the reaction, slow down the reaction and decrease the concentration of the product.

\section{Basics of Supercritical Fluids}

\section{Supercritical fluid (SCF)}

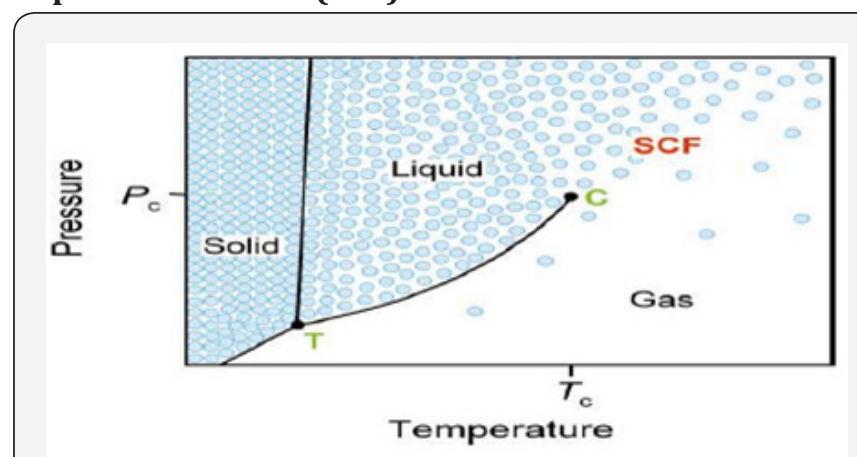

Figure 11: Schematic P-T phase diagrams for a pure component showing the supercritical fluid (SCF) region. (Adapted from Ref.9).

The triple point (T) and critical point (C) are marked. The blue circle represents the variation in density of the substance in the different regions of the phase diagram. Note that the density varies continuously between the liquid state and the gas state, providing the liquid-gas equilibrium (Figure 11).

Three different phases of the compound-solid, liquid and gas are also shown. With an increase in temperature and pressure, there comes a point when the compound shows intermediate properties between those of a pure liquid and a pure gas that is the critical point. The region beyond that critical point is the supercritical fluid.

\section{Definition}

What is supercritical fluids?: When the temperature and pressure of a one-component fluid exceed its critical temperature and pressure, it is said to be supercritical fluids. It is neither liquid, nor gas as shown in the diagram and that give no any clue to predict the properties of supercritical fluids (SCF). Therefore, supercritical fluids are influencing the chemical processes in many ways. They can be used as solvents since they have unique properties to become as liquids and as gases together. Furthermore, these properties offer distinct advantages such as:

\section{Gas-Expanded liquids (GXLs)}

A Gas-expanded liquid (GXL) is mixed solvent composed of a compressible gas such as carbon dioxide or ethane dissolved in an organic solvent. In other words, it is the ability of the gas to expand the liquid (solvent) and improve the physical and chemical properties of the liquid (Figure $3 \mathrm{~b}$ ). It is considered a magical chemical due to their unique properties. As Jessop's group mentioned, (GXLs) are the promising alternative media for performing synthesis, and separation [7] (Figure 12).

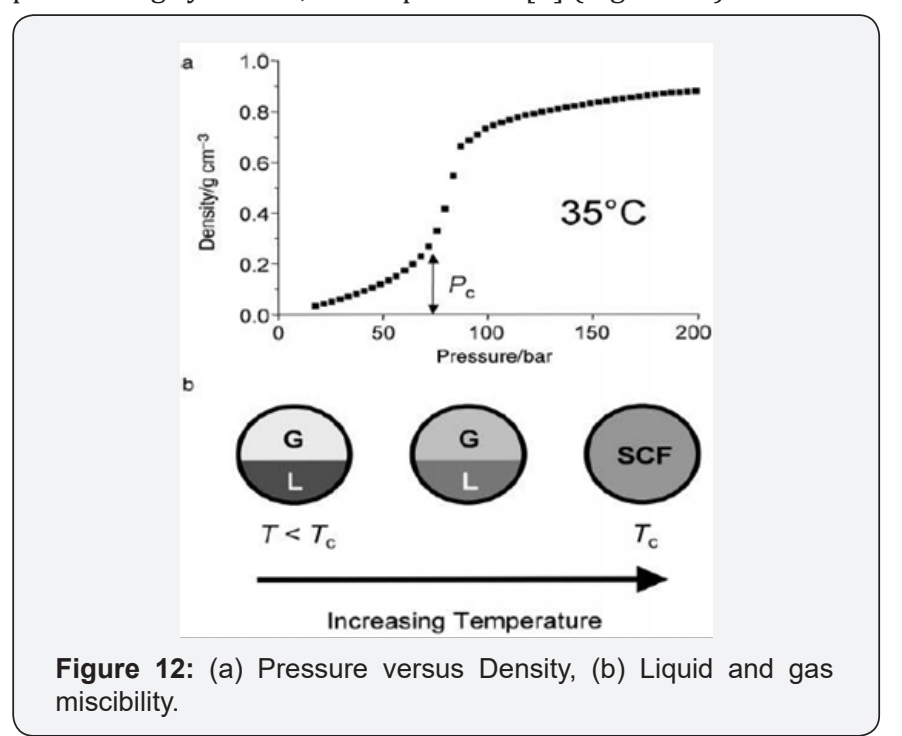

Figure 12a Graph showing the variation in density for pure $\mathrm{CO} 2$ at $35^{\circ} \mathrm{C}$. At this temperature (i.e., close to Tc for $\mathrm{CO}_{2}$ ) there is a rapid but continuous increase in density near the critical pressure (Pc). (b) Schematic representation of the change from liquid+gas equilibrium $(\mathrm{T}<\mathrm{Tc}$ ) to supercritical fluid $(\mathrm{T} \geq \mathrm{Tc})$ conditions as a substance is heated above its critical temperature at a pressure in excess of Pc.

\section{Supercritical carbon dioxide $\left(\mathrm{SCCO}_{2}\right)$}

Supercritical carbon dioxide $\left(\mathrm{SCCO}_{2}\right)$, at $\left(\mathrm{T}_{\mathrm{c}}=304.1 \mathrm{~K}\right.$, $\mathrm{P}_{\mathrm{c}}=7.38 \mathrm{MPa}$ ), is the promising green solvent due to it is unique 
property and behavior such as non-flammable, inexpensive, and environmentally friendly and safe. It is also available abundantly. Many researchers have shown that carbon dioxide has a high potential as a solvent for many organic compounds and widely used for practical application [8] (Figure 13).

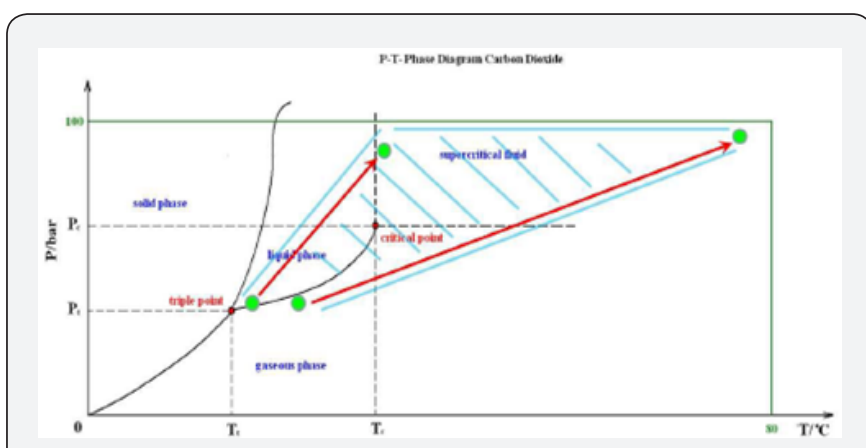

Figure 13: P-T Phases Diagram Carbon Dioxide.

This diagram shows the P-T phase diagram with the region of supercritical Carbon dioxide properties $\left(\mathrm{T}_{\mathrm{c}}=304.1 \mathrm{~K}\right.$, $\mathrm{P}_{\mathrm{c}}=7.38 \mathrm{MPa}$ ).

Applications of supercritical carbon dioxide: The application of supercritical carbon dioxide $\left(\mathrm{scCO}_{2}\right)$ as a solvent has been on the rise. This is because of its low toxicity and its usage in compound extraction. Further, it has a higher stability for the different process reactions and need low temperatures for any process to compare with other solvents [9]. Perhaps the most common use of supercritical carbon dioxide $\mathrm{scCO}_{2}$ is in the coffee industry. In addition, the application of supercritical carbon dioxide has found its way into the pharmaceutical industry. It is used in the production of micro-and nano-scale particles [1]. Polymerization is another area that makes use of supercritical carbon dioxide. It has proved to be a good nonpolar solvent for organic compounds such as alkanes, alkenes, aromatics, and alcohols which can easily dissolve in supercritical carbon dioxide. It is seen that polar molecules such as sugars and other inorganic salts are insoluble but by increasing the pressure of supercritical fluid, the dissolving power can be improved [8]. Many studies noted that advantages of supercritical carbon dioxide are attracting industries due to that they are inexpensive, non-toxic, can be recycled, and has easily accessible critical conditions. Also, supercritical carbon dioxide $\mathrm{scCO}_{2}$ processing of polymer uses the liquid-like densities and gas-like mass transfer properties of dense gases (DGs) to provide more controlled and more efficient methods of polymers preparation. Also provides an environmentally benign method for creating polymer compared with conventional processing that increase the environmental threat by emitting Volatile Organic Compounds VOC's and other polluted compounds into the atmosphere.

\section{Supercritical fluids applications:}

a. Supercritical Extraction

b. Particle formation
1. Particles from Gas-Saturated Solution (PGSS)

2. Gas Anti solvent (GAS)

3. Precipitation with Compressed Ant solvent (PCA)

4. Solution-Enhanced Dispersion by Supercritical Fluids (SEDS)

5. Depressurization of an Expanded Liquid Organic Solution (DELOS).

c. Supercritical Fluid Chromatography

d. Separations and crystallization

Synthesis and processing: The review focuses on recent advance in polymer synthesis and processing using liquid and supercritical carbon dioxide $\mathrm{scCO}_{2}$. The synthetic techniques discussed include homogeneous solution polymerization and heterogeneous and the variable solvents strength associated with supercritical fluids have been used in areas such as polymer fractionation and polymer extraction. Furthermore, the unique physical properties associated with supercritical fluids give rise to a range of possibilities for the chemical materials. Not only in polymer science but also in organic synthesis, heterogeneous and homogeneous catalysts. In the case supercritical carbon dioxide $\left(\mathrm{SCCO}_{2}\right)$, there are chemical, environmental, and economic benefits that will be captured from its use as a solvent.

In addition, supercritical carbon dioxide technology has several advantages over conventional solvents as a reaction media [3]. The unique property of supercritical fluids observed from different SCF experiments maybe used to manipulate chemical reactions to make them more efficient or specific. In addition, SCF has a great miscibility which might change heterogeneous reactions and become homogenous reactions and the yield can be greatly improved [3]. Furthermore, it is stated that using $\mathrm{scCO}_{2}$ as a reaction media will change the properties of the reaction and enhance the reaction rate as well as the yield.

\section{Separation}

Beside the well-known environmental aspects of supercritical carbon dioxide $\left(\mathrm{SCCO}_{2}\right)$, it is also advantageous in the separation process as solvent due to its preferable critical properties $(\mathrm{Tc}=304.1 \mathrm{~K}, \mathrm{Pc}=7.38 \mathrm{MPa})$ [10]. Using supercritical fluid to separate mixture compounds is an interesting technique due to not tend to leave any toxic residuals or cause potential thermal degradation of the product [10]. On the other hand, conventional solvents where used for separation purpose are environmentally dangerous and create chemical waste that supporting the phenomenon of greenhouse gases such as volatile organic solvents (VOC).

\section{Extraction}

Extraction by Supercritical Fluids gives clean and efficient technique compared to other techniques. Extraction by supercritical fluids (SFE) technologies will enable solutes 
to dissolve in the supercritical carbon dioxide $\left(\mathrm{scCO}_{2}\right)$ and precipitated by reducing the pressure of the fluid phase. The supercritical carbon dioxide is then expanded to remove the solutes, and the gas is recycled for repeated use [3]. Decaffeinated coffee and hop extracts are good examples for the application of extraction by using supercritical $\mathrm{CO}_{2}$. However, BPA solubility in supercritical carbon dioxide will play an important role in the BPA extraction process since it is barely soluble in $\mathrm{SCCO}_{2}$ and become easy to be extracted (Figure 14).

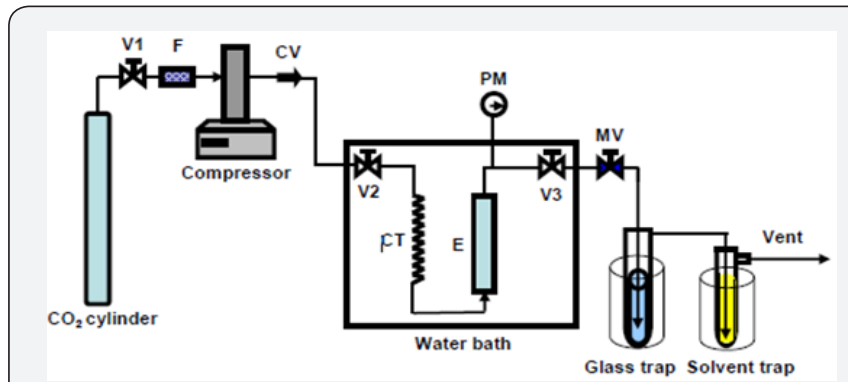

Figure 14: Schematic diagram of SCF extraction. V1, V2, V3: stopping valve; F: filter; CV: check valve, CT: coiling tube; $\mathrm{E}$ : extraction vessel; PM: pressure meter; MV: micro-metering valve.

\section{Supercritical fluids reaction}

There is no attention been given to this topic to classify the physical and chemical states of the supercritical carbon dioxide as a reaction media to produce Bisphenol A-BPA. The unique properties of supercritical fluids observed from different SFE experiments used to make the reaction more efficient has been determined to be used as a reaction media for BPA. In addition, a study made by Chen et. al. showed that using $\mathrm{ScCO}_{2}$ as a reaction media will enhance the reaction rate and yield even though Liu group mentioned that BPA was insoluble in supercritical $\mathrm{CO}_{2}$. Therefore, the study will focus on the chemical reaction of supercritical carbon dioxide.

\section{Acetone}

Acetone is an organic compound. Colourless, flammable liquid, and the simplest example of ketone with formula of $\left(\mathrm{CH}_{3}\right)_{2} \mathrm{CO}$. As Gates et al mentioned, the reaction of acetone with phenol is too slow in the absence of catalyst, but in the presence of acid resin, it proceeds with the formation of BPA. From the theoretical viewpoint, acetone with $\mathrm{SCCO}_{2}$, solubility of acetone in $\mathrm{SCCO}_{2}$ was increased with pressure and temperature [11]. Many papers showed that the solubility of acetone in supercritical carbon dioxide increased based on the condition of pressure and temperature [12]. However, diffusivity of the acetone affected by temperature, as the temperature increased the diffusion coefficient of acetone in $\mathrm{SCCO}_{2}$ increased at lower pressure [12]. Therefore, both temperature and pressure have significant effects on the diffusivity and solubility equilibrium [9]. Although acetone chemically instable, it is very obvious that acetone is soluble in $\mathrm{SCCO}_{2}$ under certain conditions which will help to get rid of the undesired products forms by AcetoneAcetone Reaction (Figure 15 \& 16).
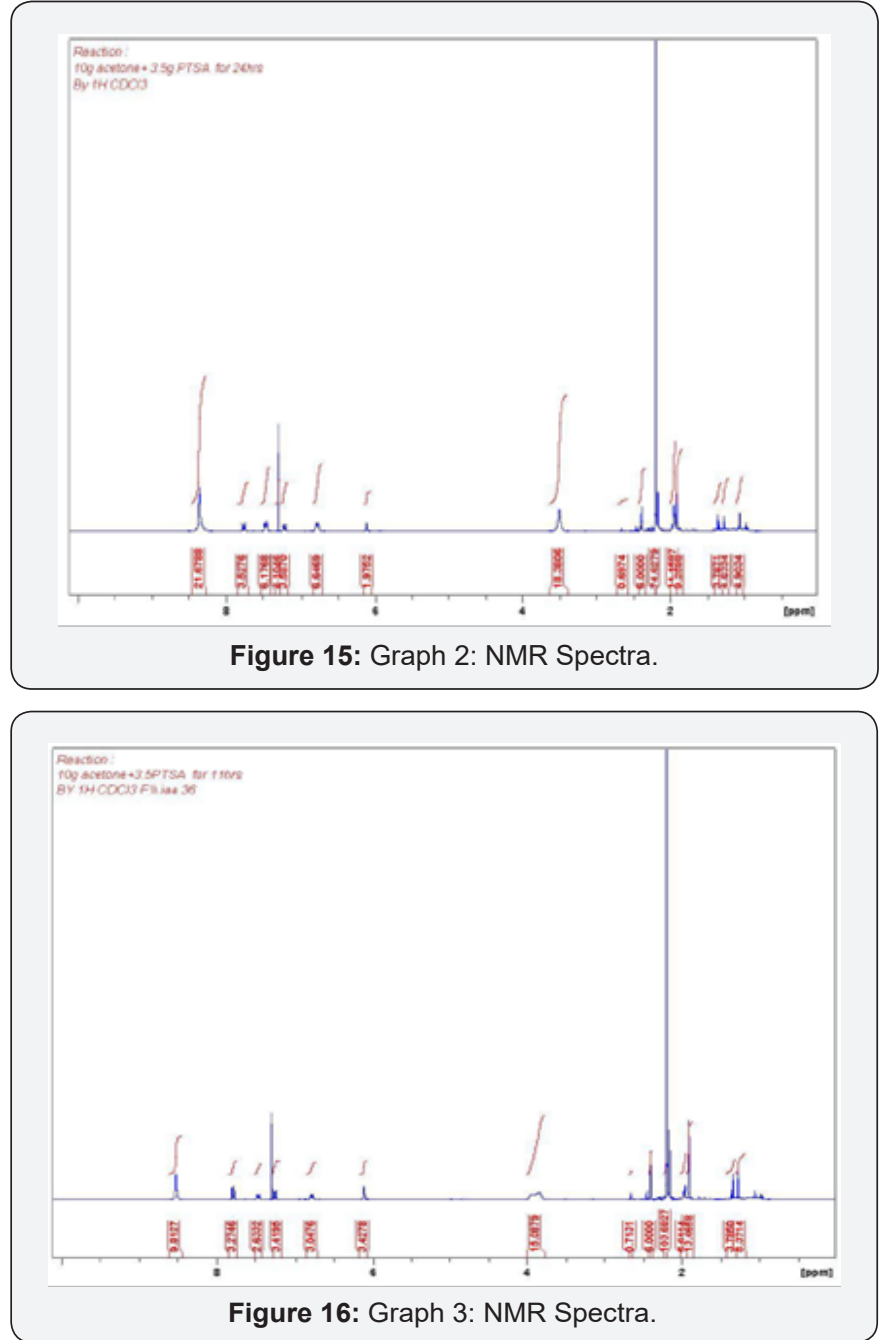

The analysis by NMR instrument shows that there are several products made by the reaction with small percentage but the main by-product is Mesityl Oxide which is detected by the analysis. It is an important by-product in this reaction because of it's high reactivity with other reactants. The study also focuses on the product as one of the research goals.

\section{Phenol}

Phenol is a chemical compound consist of hydroxyl group $(\mathrm{OH})$ connected to an aromatic hydrocarbon group. Phenol has strong acidity due to the aromatic ring. Its chemical formula is $\mathrm{C} 6 \mathrm{H} 5 \mathrm{OH}$. The chemical properties of phenol are unique due to the presence of hydroxyl group and an aromatic ring which are complementary to each other in facilitating both electrophilic and nucleophilic type of reaction (BPA reaction is electrophilic) In addition, phenol has an extremely high reactivity of its ring toward electrophilic substitution and assists its acid catalyzed reaction with acetone leading to phenolic resin. Phenol can be produced industrially with acetone by the cumene-to-phenol process, there are more than $97 \%$ of phenol produced by this process which reported by Hock and Lang. The cumene-tophenol process was developed on a commercial scale by Distillers 
Co. in Great Britain and Hercules Powder Company in USA. The first production plant was commenced in 1952 in Shawinigan, Canada. Today several licensors offer their technology such as KBR or UOP. Although all these processes use the principle of Hock synthesis, there are differences in the design and operation of the reaction units and distillation unit (Figure $17 \& 18$ ).
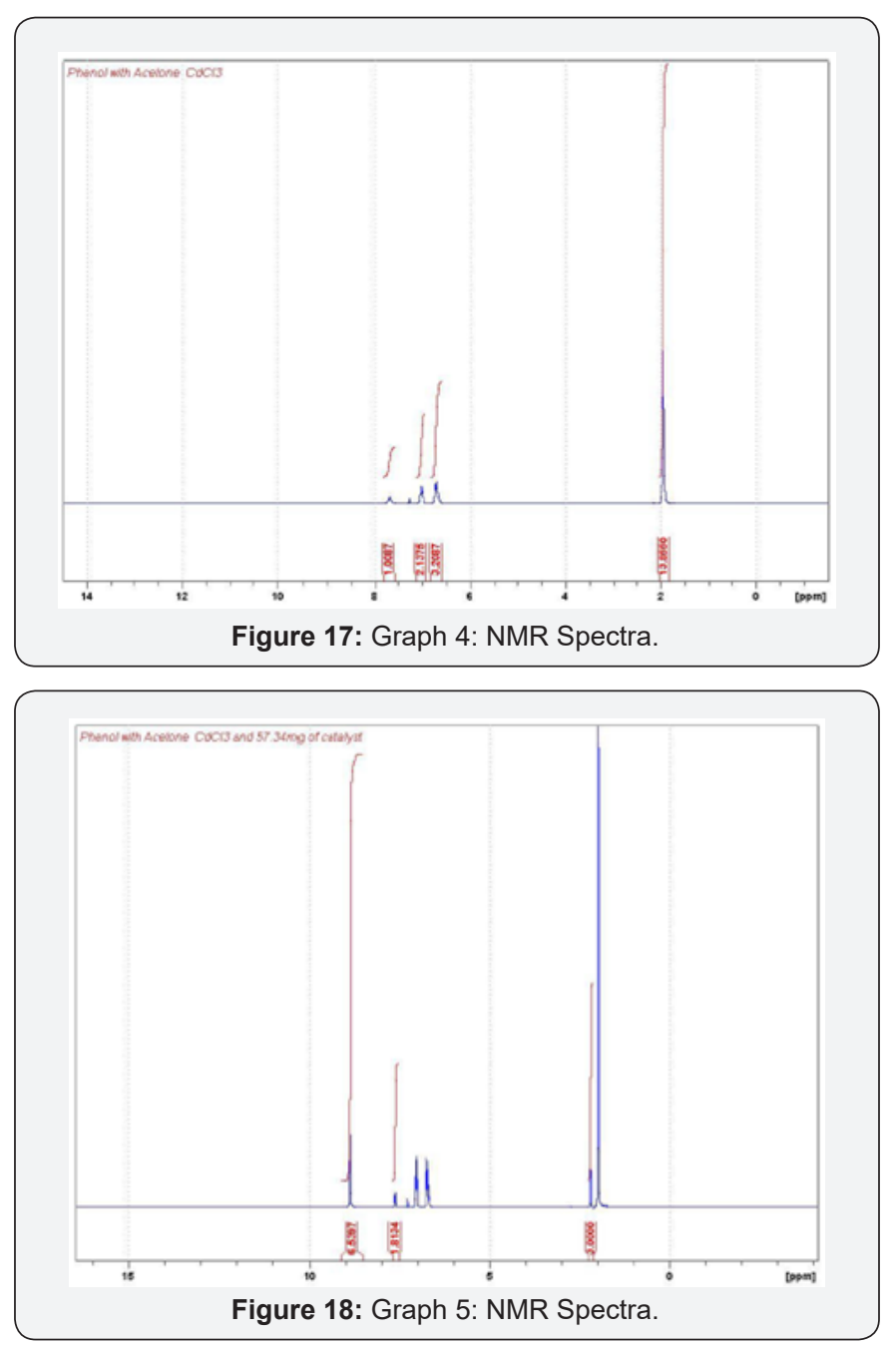

The analysis made by NMR Spectrum for Phenol and Acetone in the presence of catalyst which gives BPA. The Spectra showed the development of the product while the reactant disappeared.

\section{Solubility}

Solubility is a crude property used for determining the identity of an organic compound. It can be defined as the amount of solute that dissolves in a given amount of solvent at a definite temperature to yield a solution that is saturated in nature. However, solubility is affected by several factors such as pressure, temperature, and nature of solute and solvent [13]. Many studies on solubility in supercritical fluids have grown drastically in recent years. This due to the large range of applications of supercritical fluids in the fields of pharmaceutical, food, environmental, and industrial process. The fact that the properties of supercritical fluid can be varied from gas-like to liquid-like values by changing the pressure makes it very interesting and useful. In addition, solubility is the most important criterion affecting the efficacy of most of the supercritical fluid processes [13]. It has a direct impact on the rate, yield, design, and economy of the process, depending on the process of interest, high solubility or low solubility may be desired, for instance, high solubility is required to manufacture particle. In the case of BPA synthesis, solubility of reagents in supercritical carbon dioxide is important to accelerate the rate of reaction and enhance the reaction between acetone and phenol, thus elimination of undesired products will be achieved [13].

$\delta=\left(\frac{\Delta E}{\Delta V}\right)=T(\partial E / \partial T)-P$

Where,

$\mathrm{T}$ : is the temperature

$\mathrm{P}:$ is the pressure

\section{$\delta$ : Solubility parameter}

$\mathrm{E}$ : is the internal energy, and $\mathrm{v}$ is the molar volume.

Several studies were able to observe that an increase in temperature of certain compounds could remove the gas-liquid boundary. In the reaction of phenol with acetone, solubility improves as the difference between solubility parameter of the solute and solvents decrease [14]. The effect of Temperature and pressure will change the solubility mixture. However, the problem of selectivity of the reaction of acetone with phenol to form BPA has not been solved and the consumption of phenol and acetone still differs from the stoichiometric data.

\section{Manufacturing}

The most industrially used process for making BPA are acetone-phenol process, in homogenous and heterogeneous catalysts. Considering the costs involved and the net advantages the heterogeneous catalysts offers, the resin-catalyzed process is preferred and it has been improved continuously. A process which considers reacting acetone with very little excess phenol (1:4 or 1: 10) was reported by Azam and his group [15]. The reaction of this process consists of two steps. In the first step, the acetone is reacted with very little excess phenol in the presence of a resin catalyst to convert $20-60 \%$ of acetone. In the second step, the reaction mixture from the first step will be reacted in the presence of hydrochloric acid as a catalyst. Although there were different processes using alternative feeds, by-products still come out with the product mixture. Thus Purification is required.

\section{Purification}

The current process used to produce BPA influences the composition of the mixture from the reactor. It is still expected to contain phenol, acid catalyst (unless an acid ion- exchange resin is used), water, BPA, by-products, and some acetone. For example, a crude product stream consisted of 40\% BPA, 36.2\% o, p-isomer, $1.1 \%$ o, o-isomer, $14.2 \%$ phenol, $3.5 \%$ Chroman, 
$0.05 \%$ flavan and $12 \%$ of undefined materials. However, the composition of the BPA unusually available on the market is $95 \%$ BPA, $3 \%$ Triphenol, $1 \%$ chromanes, less than $1 \%$ o, p-isomer, and very small percentage of others. However, the BPA production is continuously improving by using different purification methods or even by changing small differences in the operating condition which may be have considerable effect on the process. Evaporation and Colum chromatography have been used in the current experiment for more purification [16-18].

Purification processes: Bisphenol A separation can be done in different ways such as evaporation, crystallization, filtration, and centrifugation. Vacuum distillation was used in this experiment to distill out the phenol at certain conditions. More purification was applied on the crude BPA to remove all undesired materials by using Colum chromatography.

Based upon these initial observations, plans were made to use dry column chromatography on the crude reaction product (tar residue) to separate the suspected phenol product from the reaction mixture and unequivocally identify that material as phenol using TLC, NMR, FTIR, and GC/MS techniques.

Colum chromatography: In column chromatography, the stationary phase, a solid adsorbent, is placed in vertical glass usually column. The mobile phase, a liquid, is added to the top and flows down through the column by either gravity or external pressure. Column chromatography is generally used as a purification technique to isolated desired compounds from a mixture [19].

The mixture to be analyzed by column chromatography is placed inside the top of the column. The liquid solvent (the eluent) is passed through the column by gravity or by the application of the air pressure. Because of the different components in the mixture have different interactions with the stationary and mobile phases, they will be carried along with the mobile phase to varying degrees and a separation will be achieved. The elutants or individual components are collected as the solvent drips from the bottom of the column. There are two categories for column chromatography separation:

a. Gravity column chromatography, when the solvent is forced down the column by gravity.

b. Flash chromatography, when the solvent is forced down the column by positive air pressure.

Procedure for dry column chromatography of crude reaction product: A solution of tar residue (crude product) from the reaction, dry silica gel powder (column chromatography grade-250 mesh) until all of the solution was absorbed into the silica powder which was then air-dried to produce a free flowing powder containing crude reaction product. This powder was added to the top of a dry column of silica gel (1" diameter \& 20 " length) in preparation for preliminary cleanup of the reaction mixture. The column was elute with a total amount of $100 \mathrm{ml}$ dichloro form methane (DCM) and methanol (meOH), producing an initial band of product that eluted from the column and was collected in labelled beaker 1. After the DCM elution completed, the collected amount of product evaporated immediately on a rotary evaporator to make sure that sufficient product was isolated for unequivocal identification of the phenol component. An estimate of the yield from the reaction was made at that time; however, TLC analysis indicated the presence of the phenol component (estimated by darkness intensity of product spot). Two solvents mixture were tested:

1) Aceto nitrile $\mathrm{CH}_{3} \mathrm{CN}$ and Dichloromethane $\mathrm{DCM} \mathrm{CH} \mathrm{CH}_{12}$

2) $\mathrm{MeOH}$ and Dichloroformmethane $\mathrm{DCM} \mathrm{CH}_{2} \mathrm{C}_{12}$

Up to this time, however, the isolation of phenol component was only suspected from TLC evidence, not proven by unequivocal means. Analysis of the reaction products using GC-MS and LCMS, confirmed the presence of phenol but NMR technique was used in this reaction to detect the structure of bisphenol A and phenol as un reacted compound (Figure 19) [20-23].

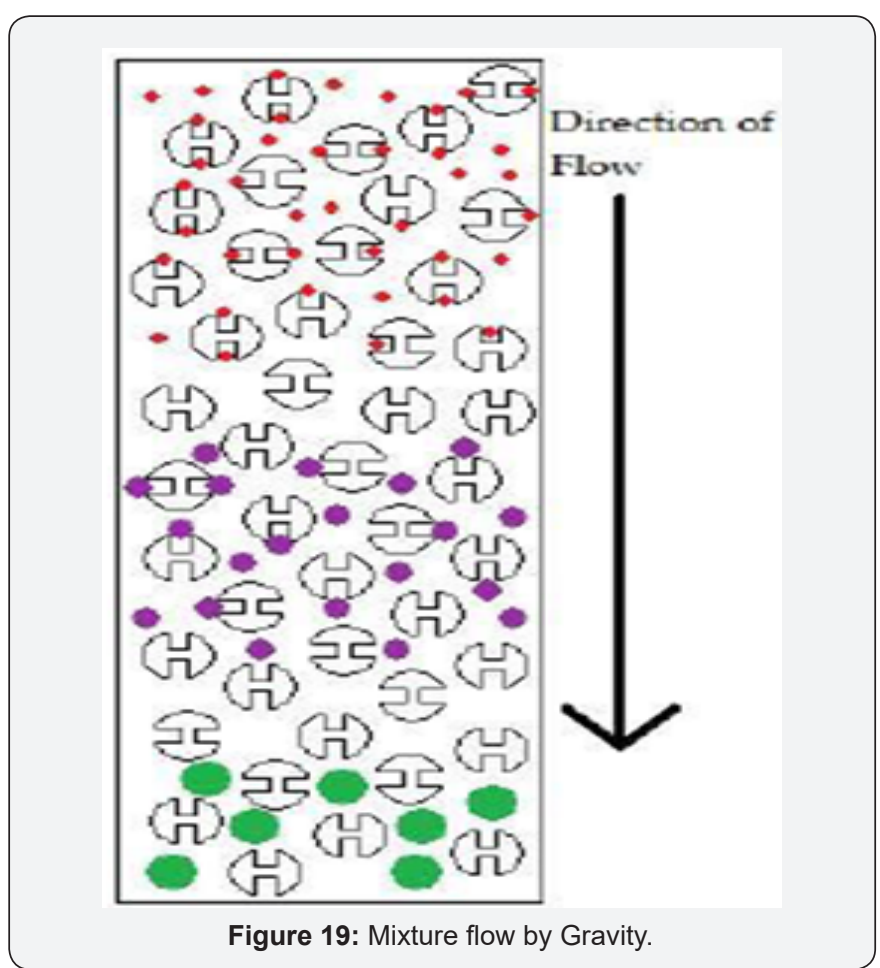

The absorbent: The absorbent used in the experiment is silica gel $\left(\mathrm{SiO}_{2}\right)$ which is solid 130-400.

The solvent: The polarity of the solvent which is passed through the column affects the relative rates at which compounds move through the column. Polar solvents can be more effectively compete with polar molecules of a mixture for the polar sites on the adsorbent surface and will also better solvate the polar constituents. Consequently, a highly polar solvent will move even highly polar molecules rapidly through the column. If the solvent is too polar, movement becomes too rapid, and little or no separation of the components of the mixture will result, but 
if the solvent is not polar enough, no compounds will elute from the column. Proper choice of an eluting solvent is essential for better separation [24-26].

Sand: It was recommended to use sand in the column to enhance separation and stabilize the stationary phase in the column.

Thin-layer chromatography (TLC): It is a method used by organic chemistry labs and the most common method. It is generally used to analyze and determine the system for a column chromatography separation.

Thin-layer Chromatography (TLC) analysis is to determine the components present within the reaction mixture. TLC analysis (multiple elutions using Methanol/Dichloromethane) indicates that the reaction mixture isolated from the product contained a multitude by its Rf value in comparison to the Rf value obtained for authentic phenol. Short-wavelength UV light was used to visualize the otherwise colorless spots on TLC, which appeared as a dark shadows against a green fluorescent background. Authentic phenol was spotted alongside the reaction product showing a spot exhibiting Rf identical with that attribute to the phenol component in the reaction mixture. TLC obtained for crude product isolated from reaction mixture showed a sizeable spot coinciding in $\mathrm{Rf}$ with the Rf value exhibited by an authentic phenol spot. Based upon these initial observations, plans were made to use dry column chromatography on the crude reaction product (tar residue) to separate the suspected phenol product from the reaction mixture and unequivocally identify that material as phenol using TL [27].

\section{Physical properties}

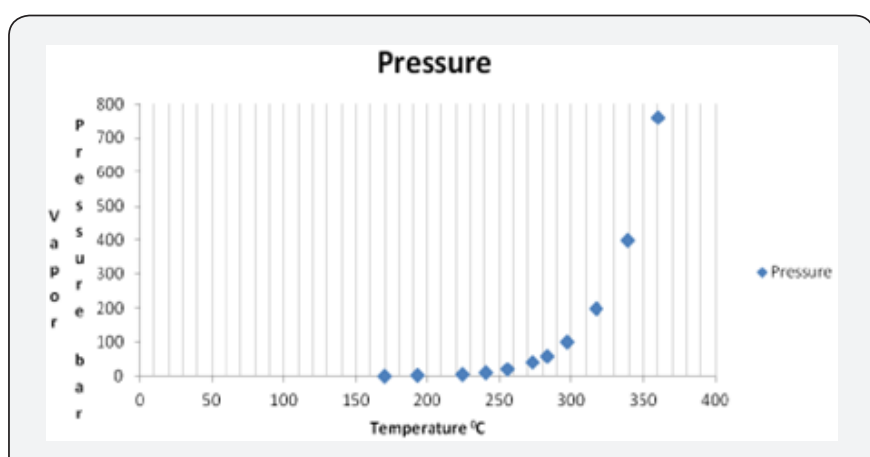

Figure 20: Vapor Pressure v/s. Temperature.

Bisphenol A is a white crystalline solid, appearing like small white to light brown flakes or powder, with mild phenolic odor, moderately high in water solubility $\left(120 \mathrm{mg} / \mathrm{l}\right.$ at $\left.25{ }^{\circ} \mathrm{C}\right)$. It is specific gravity is given as 1.195 at $25^{\circ} \mathrm{C}$. It is vapor pressure is $\left(5.32^{*} 10^{-5} \mathrm{pa} \mathrm{m}^{3} \mathrm{~mol}^{-1}\right.$. It is melt at $157^{\circ} \mathrm{C}$. It should be emphasized that the vapor pressure considerably increases at increasing temperature. Table 7: Bisphenol A Properties (Figure 20).

The graph shows the relationship between the vapor pressure of BPA with temperature. The pressure will be increased with the increasing temperature; therefore, the temperature needs to be as low as possible. It is recommended that the preferable temperature for process production ranged between $50-200{ }^{\circ} \mathrm{C}$ (Figure 21).

Table 8: Bisphenol A Properties.

\begin{tabular}{|c|c|c|}
\hline Compound & Bisphenol A & References \\
\hline Molecular formula & $\mathrm{C}_{15} \mathrm{H}_{16} \mathrm{O}_{2}$ & \\
\hline $\begin{array}{c}\text { Molecular mass (g/ } \\
\text { mol) }\end{array}$ & 228.31 & HSDB,2000 \\
\hline $\begin{array}{c}\text { Melting point (0C) } \\
\begin{array}{c}\text { Decomposition point } \\
\text { (0C) }\end{array}\end{array}$ & $153-157$ & Temanord,1996 \\
\hline Pressure Vapor (pa) & $225^{\circ} \mathrm{C} 5.32-4.110-7$ & INCLID,1996 \\
\hline & $170{ }^{\circ} \mathrm{C} 27$ & HSDB; 2000 \\
\hline $\begin{array}{c}\text { Solubility H2O }(25 \\
\left.{ }^{\circ} \mathrm{C} ; \mathrm{mg} / \mathrm{l}\right)\end{array}$ & $120-300$ & \\
\hline & 301 & \\
\hline
\end{tabular}

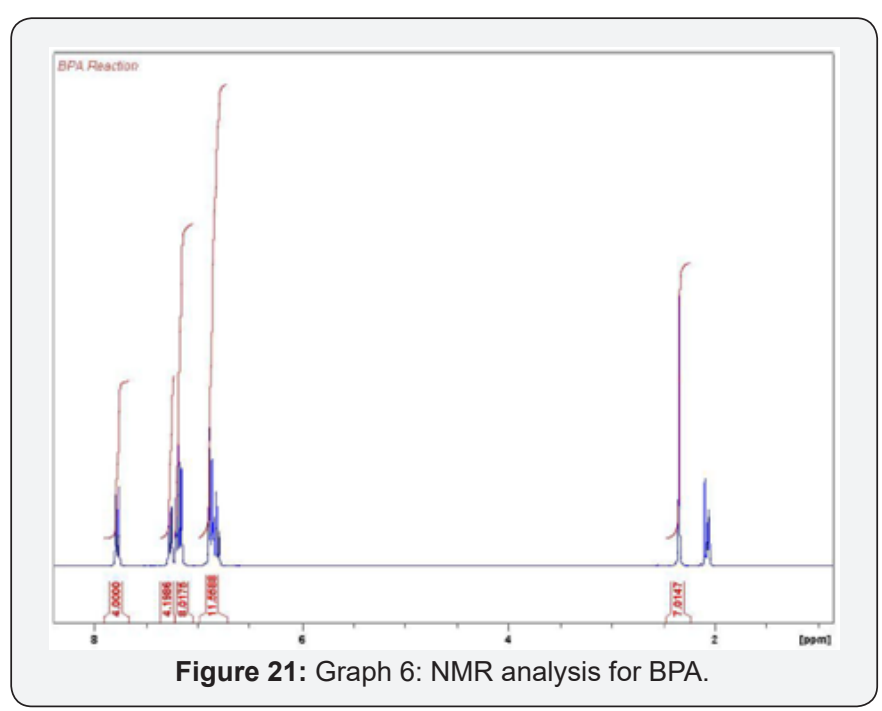

\section{Apparatus}

A liquid chromatography system consisting of binary pump (Spectra Physics (SP), P2000 LC) with helium degassing kit was used. The data-jet integrator was connected via Lab net to a personal computer with SP Winner for Windows software. The fluorescence detector was from Perkin Elmer (PE LS 40). The ultraviolet detector was from SP (UV 2000), with scanning function on eluting peaks for the second and third confirmation options [28]. High Pressure Liquid Chromatography (HPLC) will be used to analyze and identify the desired and undesired products.

\section{Chromatography conditions}

An injection system with a $50 \mu \mathrm{L}$ loop was used. Fluorescence Detector. Settings: extraction wavelength, $225 \mathrm{~nm}$ and emission wavelength, $305 \mathrm{~nm}$. 
Ultraviolet Detector, The wavelength was set at $225 \mathrm{~nm}$ (only for second confirmation option); scanning between 190 and $340 \mathrm{~nm}$ (third confirmation option).

Column, Length, $15 \mathrm{~nm}$; internal diameter, $4.6 \mathrm{~mm}$; packing, $5 \mu$ Spherisorb ODS2. Elution Program for Gradient Method. Gradient elution consisted of a 2-min isocratic elution with aceto nitrile-water (30:70); an 18-min linear gradient to $80 \%$ aceto nitrile, a 3-min linear Gradient to $100 \%$ aceto nitrile, and a 2 -min isocratic elution at $100 \%$ aceto nitrile [29].

\section{NMR}

\section{What is NMR?}

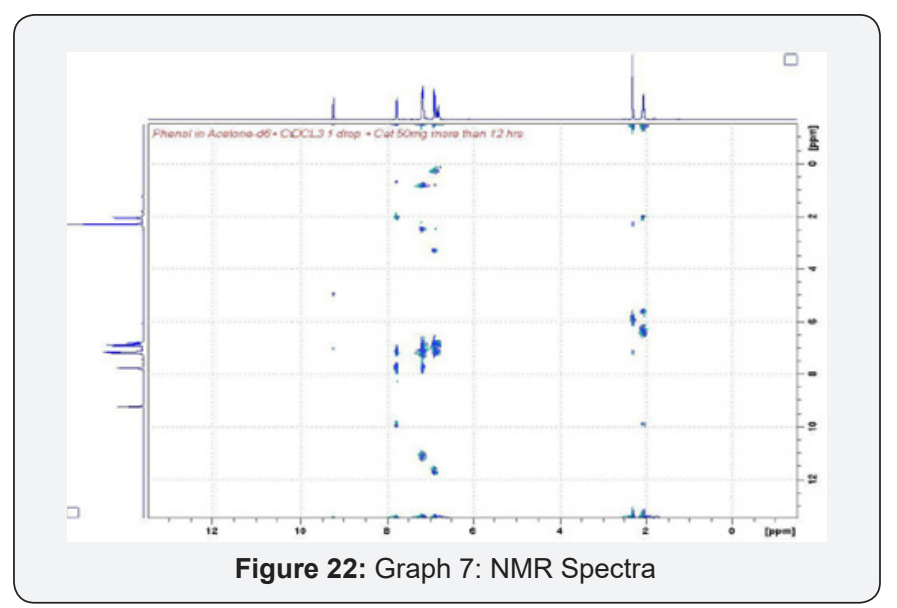

It is Nuclear magnetic resonance, or NMR as it is abbreviated by scientists, is phenomenon which occurs when the nuclei of certain atoms are immersed in a static magnetic field. Most of the matter that can be examined with NMR is composed of molecules which are composed of atoms (Figure 22 \& 23) (Table 7).

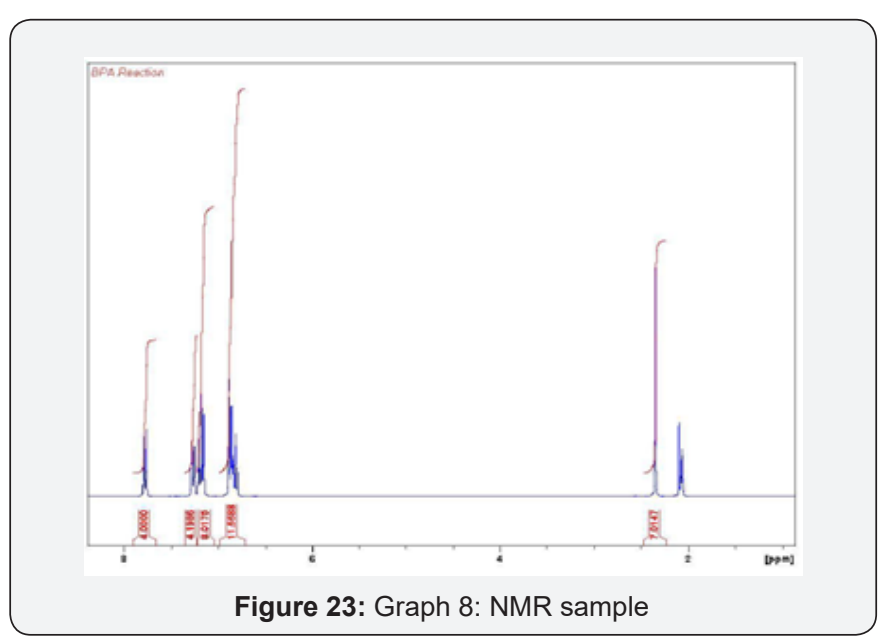

The graph presents the starting material (phenol) disappearance through the reaction time. Phenol protons (phenol A \& Phenol B) decreased while the final product will appear slowly during the reaction until BPA completely formed (Figure $24 \& 25$ ).

The curve shows the reaction of phenol during the reaction.

Table 7: Integration Table.

\begin{tabular}{|c|c|c|c|c|c|c|c|}
\hline Time & A(Phenol) & B(Phenol) & C(BPA) & D(BPA) & (C+D)/8 & (A+B)/5 & Ratio \\
\hline 0 & 4 & 4 & 0 & 0 & 0 & 1.6 & 0 \\
\hline 2 & 3.96 & 5.77 & 0.159 & 0.18 & 0.042375 & 1.946 & 0.021775 \\
\hline 4 & 2.61 & 3.66 & 0.407 & 0.403 & 0.10125 & 1.254 & 0.080742 \\
\hline 6 & 2.57 & 3.54 & 0.262 & 0.278 & 0.0675 & 1.222 & 0.055237 \\
\hline 8 & 1 & 1.455 & 0.2029 & 0.2043 & 0.0509 & 0.491 & 0.103666 \\
\hline 10 & 1.0208 & 1.424 & 0.021 & 0.0109 & 0.003988 & 0.48896 & 0.008155 \\
\hline
\end{tabular}

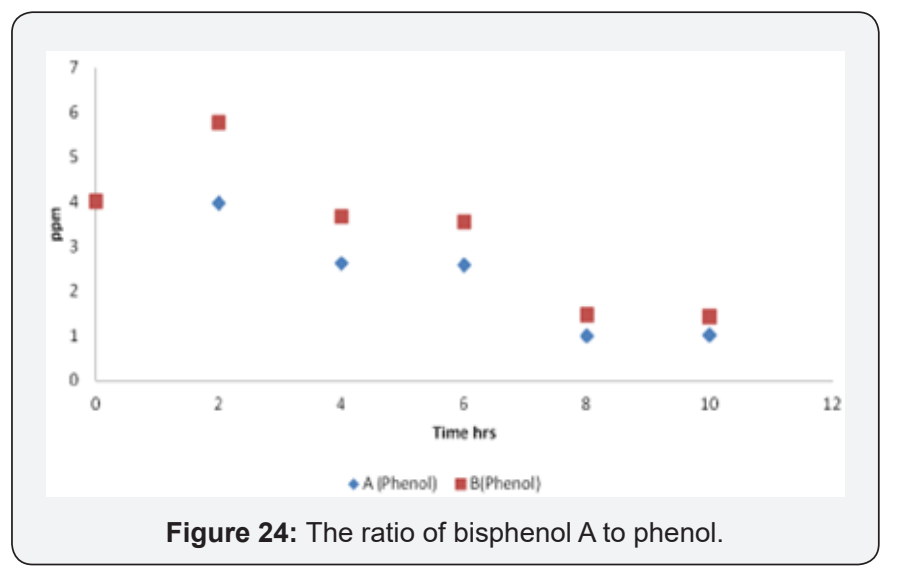

\section{HNMR-Spectroscopy}

Spectroscopy is the study of the interaction of electromagnetic radiation with matter. Nuclear magnetic resonance spectroscopy is the use of the NMR phenomenon to study physical, chemical and biological properties of matter. As a consequence, NMR spectroscopy finds applications in several areas of science. NMR spectroscopy is routinely used by chemists to study chemical structure using simple one-dimensional techniques. Twodimensional techniques are used to determine the structure of more complicated molecules. These techniques are replacing $\mathrm{X}$-ray crystallography for the determination of protein structure also. Furthermore, the versatility of NMR makes it pervasive in the sciences. This is just a basic principle necessary to begin using NMR Spectroscopy. NMR Spectroscopy will be a useful technique to be used to measure the amount of BPA produced [30].

The experiment will be carried out in the chemistry lab to apply the reaction and measure all the factors, where other techniques such as HNMR, CC, and TLC will be explained in the following sections. 


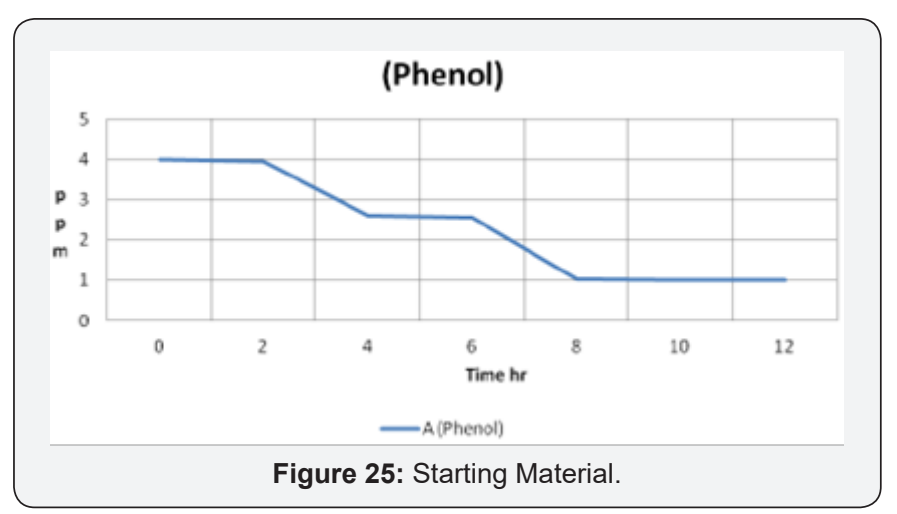

Reactor loading and set-up

1) Put on protective clothing, lab coat, goggles, and gloves. All the steps were performed in the fume hood.

2) Weigh the desired quantity of catalyst (10\%) in a clean measuring dish.

3) Transfer the catalyst into the flask.

4) Weigh the desired quantities of phenol and acetone (4.7g or $23.5 \mathrm{~g}$ of phenol and $0.58 \mathrm{~g}$ or $2.90 \mathrm{~g}$ of acetone).

5) Add the measured quantities of reagents to the reactor.

6) Turn on cooling water to condenser

7) Turn on Magnetic stirrer

8) Turn on the heat and adjust the thermostat for the desired temperature.

\section{NMR tube reaction}

1) Weigh a clean, empty NMR tube.

2) Weigh $0.05 \mathrm{~g}$ of catalyst in the NMR tube. To avoid transfer losses, the desired quantity was measured directly in the tube.

3) Prepare a mixture of acetone and phenol with ration of 1:2 acetone to phenol.

4) Take $40 \mu \mathrm{l}$ of the mixture with Pipette and put them into the tube.

5) Add $600 \mu \mathrm{l}$ of deuterated chloroform to the tube

6) Cover The NMR tube and seal carefully with para film.

7) Insert the tube in the appropriate spinner and adjust the depth using the sample depth gauge.

8) Turn on the air and position the tube in the magnet.

9) Lower the tube into the magnet by turning the air off.

10) Start the spinning air.

11) When the spinning rate reaches its set value, adjust lock power and lock gain.
12) Center and then lock signal.

13) Start heating the magnet by turning on the heater, setting the temperature at $343 \mathrm{~K}$, and increase the air flow through the magnet.

14) Once the temperature is reached, shim the field, and start the routine for data acquisition.

\section{Reactor shut-down and clean up procedure}

1) Put on protective clothing, lab coat, goggles, and gloves

2) Turn off the heater.

3) Turn off the magnetic stirrer.

4) Turn off the cooling water to the condenser.

5) When reactor is at room temperature, remove the reactor from the C-Clamp.

6) Distill it out by Rotary evaporator.

7) Take the weight of the sample

8) Put the sample in the fridge at -200C

9) Then take analyze the sample.

10) Wash flask, the stopper, and the thermometer with acetone, then with water and soap, then with distilled water, and allow them to dry.

11) Rinse thoroughly the sampling syringe with acetone to remove any residuals.

\section{NMR tube reaction}

1) Remove the tube from the magnet.

2) Turn off the heater, and set the temperature back to the room temperature value.

3) When the magnet cooled down, reduce the air flow through the magnet.

\section{Summary}

The review covers important aspects and present a new way of producing a chemical/organic material, BPA, which is important as the main feedstock for polycarbonate production. However, there are gray areas among catalysts type that might be used during the reaction with high efficiency. The paper presenting a solution of using supercritical fluid as a green technology in order to produce bisphenol A (BPA), which can be used as an essential part of polycarbonate synthesis. To limit the use of conventional methods, new and reliable methods have to be applied and by using supercritical fluid technology to produce BPA as a high chemical scale production, many industrial productions will be changed. Finally, supercritical carbon dioxide has shown its applicability for food, pharmaceutical, medical, and chemical processes. The work presented in this study focused on bisphenol a processing and the purpose of this paper is to: 
1) Identify new suitable catalysts for BPA formation

2) Find appropriate reaction conditions

3) Determine the reaction conditions that significantly influence the process and explain their influence.

The analysis of experimental design shows that the interaction effect of temperature with catalyst has significant effects on BPA yields. Furthermore, the interaction effect of temperature with time has also a significant effect on the process but in the present of a strong catalyst with the optimal operating conditions for maximum yield. Whilst individual effects of time and concentration have minor impacts on the process.

\section{Future plan}

The main part of the future work will be depend on the mixture identification and impurities detection by using different analysis techniques such as TLS, GC-MS, and NMR to confirm all undesired products which formed by acetone-acetone reaction. Therefore, supercritical carbon dioxide as a new technology to purify undesired products associated with acetone reaction (Dimerization).

\section{Appendix}

\section{A. Appendix: The NMR phenomenon}

Nuclear Magnetic Resonance (NMR) spectroscopy is a method of great interest and important for the study of chemical substance. The use of pulsed Fourier transform methods with spectrum accumulation made it possible to obtain high resolution spectra (Sander and Hunter, 1993).

\section{B. The chemical shift}

Resonance occurs at slightly different frequencies for each type of proton, depending on its chemical binding and position in a molecule. This variation is caused by the cloud of electrons about each nucleus, which shields the nucleus against the magnetic field, thus requiring a slightly lower value of v0to achieve resonance than for a bare proton (Sanders and Hunter, 1993). Protons attached to or near electronegative groups such as $\mathrm{OH}, \mathrm{OR}, \mathrm{OCOR}, \mathrm{COOR}$ and halogens experience a lower density of shielding electrons and resonate at higher v0. Protons farther removed from such groups, as in hydrocarbon chains, resonate at lower v0.These variations are called chemical shifts and are commonly expressed in relation to the resonance of tetra methyl silane (TMS) as zero of reference. The total range of proton chemical shifts in organic compounds on the order of $10 \mathrm{ppm}$.

For any nucleus, the separation of chemically shifted resonances, expressed in $\mathrm{Hz}$, are proportional to B0. When expressed in ppm, as common, the chemical shifts are independent of $\mathrm{B} 0$. The electronic screening of nuclei is actually anisotropic so that the chemical shift is a directional quantity and depends on the orientation of the molecule with respect to the direction of the magnetic field. In solution, the motional averaging produces an isotropic value of the chemical shift.

\section{Nuclear coupling}

Nuclei sufficiently removed from each other do not feel the effects of the magnetic fields of the other nuclei. In this case, the local magnetic field at each nucleus is essentially equal to B0. If B0 can be made very homogeneous over the sample, the width of the resonance may be very small.

\section{Direct dipole-dipole coupling}

In most substances, protons contribute to local fields and are sufficiently numerous to have a marked effect. The $\mathrm{C}$ atoms also contribute to the local fields, but their natural abundance is very small, therefore they do not have a visible effect.

\section{E. Indirect nuclear coupling}

Magnetic nuclei may transmit information to each other concerning their spin states not only directly through space, but also through the intervening covalent bonds. This is indirect or scalar nuclear coupling, also known a J coupling. Rapid tumbling of the molecule does not reduce this interaction to zero. If the nucleus has $n$ sufficiently close, equivalently coupled spin $-1 / 2$ neighbors, its resonance will be split into $n+1$ spin states of the neighboring group of spin. Thus one neighboring spin splits the observed resonance to a doublet, two produce a 1:2:1 triple, three a 1:3:3:1 quarter, and so on. The strength of the coupling is denoted by a coupling constant $\mathrm{J}$ and is expressed in $\mathrm{Hz}$.

\section{References}

1. Reinicker RA, Gates BC (1974) "Bisphenol A Synthesis: Kinetics of the phenol-Acetone Condensation Reaction Catalyzed by Sulfonic Acid Resin" Department of Chemical Engineering, University of Delaware, Newark, Delaware Sep 20(5): 933-940.

2. European Patent Application, published with ART: 158(3) EPC, 2004.

3. Wai CM, Hunt F, Ji M, Chen X (1998) "Chemical Reaction in Supercritical Carbon Dioxide", Journal of Chemical Education 75(12): 1641-1645.

4. Griswold J, Wong SY "Phase-Equilibria of The Acetone-Methanol-Water system from 100C into The Critical Region" University of Texas, Austin, Sumposium Series 3: 18-31.

5. Jung H, Kim H, Chun D, Hong JC, Heon J, et al. (2010) "Mass Transfer limitations on fixed-bed reactor for Fischer-Tropsch synthesis" Fuel Processing Technology 91(3): 285-289.

6. Motoaki K, InoueY, Araki T, Hashimoto K (1999) "The simulated moving-bed reactor for production of Bisphenol A" Catalysis Today 48(1-2): 199-209.

7. Jessop PG, Bala Subramanian (2007) “Gas-Expanded Liquids” American Chemical Society Reviews 107(6): 1666-1694.

8. Coutsikos P, Magoulas K, Tassios D (1995) “Solubilities of Phenols in Supercritical Carbon Dioxide" Journal of Chemical Engineering Data 40(4): 953-958.

9. Kurnik RT, Creid R (1982) "Solubility of Solid Mixture in Supercritical Fluids" Fluid Phase equilibria 8(1): 93-105.

10. Benning R, Hu M, Ertunc O, Neukam J, Bielke T, et al. (2011) "Mass transfer of organic substances in supercritical carbon dioxide" International Symposium on Physical Science in Space, Journal of Physics: Conference Series 326-328: 360-365.

11. Cooper IA (1999) "Polymer Synthesis and Processing Using 
Supercritical Carbon Dioxide" Department of Chemistry, The Donnan and Robert Robinson Laboratories, University of Liverpool, Crown Street, Liverpool, UK.

12. Nishiumi H, Fujita M, Agou K (1996) "Diffusion of Acetone in Supercritical Carbon Dioxide" Chemical Engineering Laboratory, Hosei University, Koganei, Tokyo 117(1-2): 356-363.

13. Martin A, Rodriguez, Laura D, Cocero M (2011) “Solubility of Bisphenol A in Supercritical Carbon Dioxide" Journal of Chemical \& Engineering Data 56(10): 3910-3913.

14. Castro AE "Solubility of Organic Compound" De La Salle UniversityDasmarinas, Dasmarinas, Cavite.

15. Rahimi A, Farhangzadeh S (2001) "Kinetics Study of Bisphenol A Synthesis by Condensation Reaction" Department of Polymer \& Catalysts, Iran Polymer Institute, Tehran, Iran.

16. Krishnan AV, Stathis P, Permuth SF, Tokes L, Feldman D (1993) "Bisphenol A:An Estrogenic Substance is Released from Polycarbonate Flasks during Autoclaving*”, Division of Endocrinology, Stanford University School of Medicine, Stanford, California 132(6): 2279-2286.

17. Silva MSD, Raquel V, Monica B, Aguiar-Ricardo A, Teresa C (2012) "Supercritical CO2-assisted preparation of a PMMA composite membrane for bisphenol A recognition in aqueous environment" Chemical Engineering science 68(1): 94-100.

18. Kim S, Johnston KP (1987) “ Clustering in Supercritical Fluid Mixtures" AIChE Journal 33(10): 1603-1611.

19. Philippos C, Magoulas K, Tassios D (1995) "Solubilities of phenols in Supercritical Carbon Dioxide" Laboratory of Thermodynamics and Transport Phenomena, Department of Chemical Engineering, National Technical University of Athens, Greece 40(4): 953-958.
20. Stephen M, Ramone D, Mitsutoshi J, Joseph R, Saad K, et al. (1998) "Synthesis and Swelling of Poly (bisphenol A carbonate) Using Supercritical CO2" Department of Chemistry, University of North Carolina at Chapel Hill and Kenan Laboratories 31(25): 9090-9092.

21. Stankiewicz A, Multiphase Reactors; Process \& Eng. Department, Delft University of Technology, The Netherlands.

22. Harmer MA, Sun Q (2001) "Solid acid catalyst using ion-exchange resins" Applied Catalysis A: General 221(1-2): 45-62.

23. Prokop Z, Hankova L, Jerabek K (2004) "Bisphenol A synthesismodeling of industrial reactor and catalyst deactivation" Reactive \& Functional Polymers 60: 77-83.

24. Jerabek K, Odnoha J, Setinek K (1988) "Kinetics of the Synthesis of Bisphenol A" Applied Catalysis 37: 129-138.

25. Robert K, Akgerman A, Brenda, Irvin (1989) “Extraction of Phenol from water with supercritical carbon dioxide" The journal of supercritical fluids 2(2-3): 51-56.

26. Hyatt JA (1984) "Liquid and Supercritical Carbon Dioxide as Organic Solvents" Research Laboratories, Eastman Chemicals Division, Kingsport, Tennessee 49(26): 5097-5101.

27. Fornari T, Hernandez E, Reglero G (2009) "Solubility of Supercritical gases in Organic Liquids" Madrid, Spain 51(2): 115-122.

28. Yoganathan R, mammukari R, Foster NR (2009) Thesis "Polymer Processing Using Dense Gas Technology”, polymer reviews 50(2): 144177.

29. UNSW

30. United States Patent (1994) "Used of Acetone Conversion for Capacity Increase and Quality/Yield Improvement in The Bisphenol-A Reaction".

\section{Your next submission with Juniper Publishers will reach you the below assets}

- Quality Editorial service

- Swift Peer Review

- Reprints availability

- E-prints Service

- Manuscript Podcast for convenient understanding

- Global attainment for your research

- Manuscript accessibility in different formats

( Pdf, E-pub, Full Text, Audio)

- Unceasing customer service

\section{Track the below URL for one-step submission}

https://juniperpublishers.com/online-submission.php 